\title{
Extracapsular Spread in Head and Neck Carcinoma: Impact of Site and Human Papillomavirus Status
}

\author{
Jessica H. Maxwell, MD, MPH'; Robert L. Ferris, MD, PhD; William Gooding, $\mathrm{MS}^{2}$; Diana Cunningham, $\mathrm{MS}^{2}$; \\ Vikas Mehta, MD³; Seungwon Kim, MD; Eugene N. Myers, MD'; Jonas Johnson, MD'; and Simion Chiosea, MD ${ }^{4}$
}

BACKGROUND: Extracapsular spread (ECS) in cervical lymph node metastases from head and neck squamous cell carcinoma (SCC) is regarded as an adverse prognostic factor and is often used to select patients who may benefit from adjuvant therapy. The prognostic value of ECS was evaluated for patients with oropharyngeal SCC (OPC; with known p16/human papillomavirus [HPV] status) and for patients with SCC of the oral cavity (OCC). METHODS: Disease-specific survival (DSS) was assessed among SCC patients with cervical lymph node metastases ( $n=347$, including 133 patients with OPC and 214 patients with OCC). All patients were treated surgically between 1983 and 2009. ECS status was determined by pathologists at the time of initial pathologic evaluation and confirmed for this study. HPV status of patients with OPC was determined via immunohistochemistry for p16 and in situ hybridization. RESULTS: Among OCC patients, ECS was a significant, independent factor influencing DSS. For OCC patients with ECS, 3-year DSS was 45\% (95\% confidence interval [CI], 36\%-56\%); for those without ECS, 3-year DSS was $71 \%(95 \% \mathrm{Cl}, 62 \%-81 \%$; $P=.0018)$. The effect of ECS was independent of the number of positive lymph nodes as well as other clinical, pathologic, and treatment variables. Of the 133 OPC patients, 76 (57\%) were p16-positive and 57 (43\%) were p16-negative. ECS status did not correlate with DSS among p16-positive or p16-negative OPC patients. CONCLUSION: ECS was not associated with worse DSS in p16-positive or p16-negative OPC patients. Adverse prognostic value of ECS in OCC patients was confirmed. Cancer 2013;119:3302-8. ( 2013 American Cancer Society.

KEYWORDS: Extracapsular spread; HPV; p16, oropharynx; oral cavity; squamous cell carcinoma.

\section{INTRODUCTION}

Extracapsular spread (ECS) in cervical lymph node metastases from head and neck squamous cell carcinoma is regarded as a poor prognostic factor. ${ }^{1,2}$ ECS has been associated with an increased number of nodal metastases, ${ }^{3}$ regional recurrences, ${ }^{4,5}$ and distant metastases. ${ }^{4-7}$ The significance of ECS has been demonstrated in various subsites of the head and neck, including the oral cavity and larynx. ${ }^{5,8-11}$ However, the literature has not sufficiently addressed the possibility that the prognostic value of ECS may be modulated based on the site of the primary squamous cell carcinoma (SCC). The few studies that have analyzed ECS in the oropharynx have not explicitly separated this site from the oral cavity ${ }^{12,13}$ nor controlled for human papillomavirus (HPV) status, ${ }^{14}$ a well-established etiologic and prognostic factor for oropharyngeal squamous cell carcinoma (OPC). ${ }^{15-18}$ Although 1 group of investigators has examined ECS in HPV-positive OPC, ${ }^{19,20}$ the significance of ECS in HPV-negative OPC remains unclear.

Reevaluating the importance of ECS in OPC becomes even more relevant as minimally invasive surgical techniques are being used increasingly, including trans-oral robotic surgery (TORS) and laser microsurgery (TLM). With more OPC patients undergoing primary surgical therapy, prognostic markers, such as ECS, can help to identify those patients who may or may not benefit from aggressive adjuvant chemoradiation therapy (CRT). Given the distinct biology and rising incidence of HPV-positive OPC ${ }^{21,22}$ and the reemergence of primary surgical management for OPC, we evaluated the prognostic value of ECS while controlling for anatomic site (oral cavity versus oropharynx), primary treatment modality (surgery), and HPV status.

\section{PATIENTS AND METHODS}

\section{Study Population}

After approval was obtained from the Institutional Review Board of the University of Pittsburgh Medical Center, the head and neck cancer database at our institution was reviewed for cases of oropharyngeal and oral cavity SCC from 1983 to 
2009. Patients who underwent primary surgical management of the tumor underwent ipsilateral or bilateral neck dissection, or had cervical lymph node metastases were included in the study. All OPC patients had histologically proven SCC of either the base of tongue or tonsil. The availability of formalin-fixed paraffin-embedded tissue block was an additional inclusion criterion for cases of OPC. Cases were excluded if they had prior head and neck irradiation, had previous head and neck cancer, or received preoperative radiation or chemotherapy. Overall, 351 patients (137 OPC patients and 214 patients with oral cavity squamous cell carcinoma [OCC]) with cervical lymph node metastases and known ECS status were included in the study. Statistical analysis was performed on only those patients with $>2$ months of follow-up (133 of the 137 OPC patients).

\section{Clinico-pathologic Data}

Indexed clinical data included age, sex, primary tumor margin status, number of positive lymph nodes, adjuvant radiation and/or chemotherapy, status at last follow-up, and tumor-node-metastasis classification as defined by the 7th edition of the American Joint Committee on Cancer (AJCC),${ }^{23}$ and primary tumor site for OPC patients only (base of tongue or tonsil).

\section{Extracapsular Spread}

ECS of the cervical lymph node metastases removed at the initial surgery was determined from pathology reports in the electronic medical record. Pathologists from the Department of Pathology at the University of Pittsburgh Medical Center reviewed each case at the time of initial evaluation and reported on ECS. If there was no documentation regarding ECS in the pathology report, a staff pathologist (S.C.) reviewed the hematoxylin and eosin (H\&E)-stained slides and determined ECS status. ECS was classified as positive or negative based on traditional criteria. ${ }^{1}$

\section{HPV via In Situ Hybridization and P16 via Immunohistochemistry}

All 137 OPC cases were tested for HPV via in situ hybridization and p16 via immunohistochemistry (IHC). One pathologist (S.C.) reviewed the hematoxylin and eosinstained slides for each case and selected a block with adequate tumor to test. When possible, the primary tumor was analyzed. However, when the primary tumor was not available or was too small for analysis, a lymph node with metastatic disease was used to determine HPV and p16 status. To ensure the concordance of HPV/p16 status among primary tumor and lymph node metastases,
20 cases were tested for HPV/p16 on the primary tumor and the corresponding lymph node metastasis and all 20 cases had concordant results.

HPV detection via in situ hybridization was performed using probes targeting 37 distinct HPV subtypes, including $6,11,16,18,31,33,35,39,45,51$, and 52 (Y1404; Dako, Carpinteria, CA). Five-micrometer tissue sections were deparaffinized and digested with proteinase K (Roche Diagnostics, Indianapolis, IN). Cases with punctate nuclear signal were considered positive. ${ }^{24}$

For p16 analysis, $5-\mu \mathrm{m}$ sections were deparaffinized. Heat-induced epitope retrieval was then performed in a citrate buffer. Immunohistochemistry for p16 (G175405; BD Pharmingen, San Diego, CA) was performed according to the manufacturer's protocol. Cases were reviewed by a staff pathologist (S.C.) and were considered positive if $>70 \%-80 \%$ of tumor cells showed diffuse, strong cytoplasmic, and nuclear staining. ${ }^{24}$

P16 status via immunohistochemistry was used for the statistical analysis of those OPC patients included in the statistical analysis $(\mathrm{n}=133)$. Oral cavity cancer patients in our study were not tested for HPV or p16 and were assumed to be p16-negative, based on exceedingly low HPV prevalence in OCC reported in the literature. ${ }^{25}$ Furthermore, our unpublished experience at the University of Pittsburgh of 73 cases of OCC tested for HPV between 2011-2012 revealed that only 1 of the 73 tumors (1.3\%) was p16-positive.

\section{Statistical Analysis}

The primary endpoint was disease-specific survival (DSS) defined as elapsed time from date of diagnosis until death from cancer. Patients who were alive at last follow-up or had died from other causes were censored. Cross-tabulated categorical data were tested for independence using a Fisher exact test. Survival data were presented as KaplanMeier plots with annual 95\% Greenwood confidence intervals (CI). The log-rank test was used to test survival equality by ECS or HPV status. Proportional hazards modeling was applied to construct a survival model that adjusted for important confounding variables. Covariates examined for association with survival included age, sex, pack-years of smoking, tissue site (for OPC, tonsil or base of tongue), total number of positive lymph nodes, margin status, N stage, T stage, AJCC stage, adjuvant chemotherapy or radiotherapy. Only patients with $>2$ months of follow-up were included in outcome analysis. Number of positive lymph nodes was found to be important in both the OCC and OPC cohorts and the effect of ECS was adjusted in each cohort accordingly. Due to violation of 
the proportional hazards assumptions in the OCC cohort, interval censoring at 36 months was applied. Therefore, the adjusted model for OCC is strictly only applicable during the first 3 years following diagnosis.

\section{RESULTS}

\section{Study Population}

Overall, 351 patients with positive cervical lymph node metastases and known ECS status were included in this study. Of those, 137 had an oropharyngeal primary and 214 had an oral cavity primary. The median follow-up time was 8 years. Of 95 censored patients (alive or dead from other causes), 80 were followed for more than 2 years. Of the 137 OPC patients, 4 patients were excluded in the statistical analysis because they did not have $>2$ months of follow-up. Patient demographics and clinicopathologic data are provided in Table 1. Compared with patients with oral cavity SCC, OPC patients were slightly younger (3 years), less likely to be female, and more likely to present with early stage tumors (pathological T1/T2) but with advanced nodal disease. Both cohorts presented with the same mean number of positive lymph nodes. ${ }^{3,4}$ ECS positivity was similar among the 2 cohorts ( $48 \%$ for OCC versus $55 \%$ for OPC). With respect to oncologic outcome, the 3-year DSS probability for OCC patients was $58 \%$, substantially worse than the $76 \%$ DSS for OPC patients $(P=.0004$; Table 2$)$.

\section{Oropharyngeal Cancer and ECS}

The clinical characteristics for OPC patients are depicted in Table 3. The median follow-up time for our OPC patients without a recurrence was 93 months (range, 2325 months). Among OPC patients, there were 43 recurrences with 39 patients who died of their disease and 4

TABLE 1. Demographics and Clinico-pathologic Data of Patients With Squamous Cell Carcinoma Metastatic to Cervical Lymph Nodes, by Site of Primary Tumor

\begin{tabular}{|c|c|c|c|c|}
\hline Characteristic & Total & Oral Cavity & Oropharynx ${ }^{a}$ & $P$ \\
\hline All patients & $347(100)$ & $214(61)$ & $133(39)$ & \\
\hline Age, y, median (range) & $59(24-90)$ & $60(24-90)$ & $57(31-85)$ & .0815 \\
\hline Sex, \% men & 72.3 & 64.5 & 84.9 & $<.0001$ \\
\hline ECS, no. (\%) & & & & .1494 \\
\hline Positive & $191(55.0)$ & $103(48.1)$ & $53(39.8)$ & \\
\hline Negative & $156(45.0)$ & $111(51.9)$ & $80(60.2)$ & \\
\hline T stage, no. (\%) & & & & $<.0001$ \\
\hline 1 & $59(17.2)$ & $18(8.5)$ & $41(31.1)$ & \\
\hline 2 & $125(36.3)$ & $69(32.5)$ & $56(42.4)$ & \\
\hline 3 & $70(20.3)$ & $46(21.7)$ & $24(18.2)$ & \\
\hline 4 & $90(26.2)$ & 79 (37.3) & $11(8.3)$ & \\
\hline $\mathrm{N}$ stage, no. (\%) & & & & .0007 \\
\hline 1 & $120(34.6)$ & $90(42.1)$ & $30(22.6)$ & \\
\hline 2 & $211(60.8)$ & $116(54.2)$ & $95(71.4)$ & \\
\hline 3 & $16(4.6)$ & $8(3.7)$ & $8(6.0)$ & \\
\hline Positive nodes, mean (range) & $3.4(1-32)$ & $3.4(1-32)$ & $3.4(1-21)$ & .595 \\
\hline
\end{tabular}

${ }^{a}$ Oropharynx patients with $<2$ months of follow-up were excluded.

Abbreviation: ECS, extracapsular spread.

TABLE 2. Disease-Specific Survival by Tumor Site and Human Papillomavirus Status for Oropharyngeal Squamous Cell Carcinoma

\begin{tabular}{|c|c|c|c|c|}
\hline Characteristic & $\begin{array}{l}\text { Number } \\
\text { at Risk }\end{array}$ & $\begin{array}{l}\text { Probability of } \\
\text { 3-Year Survival } \\
(95 \% \mathrm{Cl})\end{array}$ & $\begin{array}{l}\text { Probability of } \\
\text { 5-Year Survival } \\
(95 \% \mathrm{Cl})\end{array}$ & $P$ \\
\hline All patients & 347 & $0.65(0.60-0.71)$ & $0.58(0.53-0.64)$ & \\
\hline Tumor site & & & & .0004 \\
\hline Oral cavity & 214 & $0.58(0.51-0.65)$ & $0.51(0.44-0.59)$ & \\
\hline Oropharynx ${ }^{a}$ & 133 & $0.76(0.69-0.84)$ & $0.70(0.62-0.78)$ & \\
\hline HPV status & & & & $<.0001$ \\
\hline p16-positive & 76 & $0.89(0.82-0.96)$ & $0.84(0.76-0.93)$ & \\
\hline p16-negative & 57 & $0.58(0.45-0.73)$ & $0.48(0.36-0.65)$ & \\
\hline
\end{tabular}

Abbreviation: HPV, human papillomavirus.

a Oropharynx patients with $<2$ months of follow-up were excluded. 
TABLE 3. Demographics and Clinico-pathologic Data of Patients with Oropharyngeal Squamous Cell Carcinoma ${ }^{a}$ by ECS status

\begin{tabular}{|c|c|c|c|}
\hline Characteristic & $\begin{array}{l}\text { ECS-Positive } \\
\quad(n=80)\end{array}$ & $\begin{array}{l}\text { ECS-Negative } \\
\qquad(n=53)\end{array}$ & $P$ \\
\hline Age, y, median (range) & $58(31-85)$ & $56(37-82)$ & .7685 \\
\hline Sex & & & .332 \\
\hline Men & $70(87.5)$ & $43(81.1)$ & \\
\hline Women & $10(12.5)$ & $10(18.9)$ & \\
\hline Smoking status ${ }^{\mathrm{b}}$ & & & .101 \\
\hline Never-smoker & $17(27.9)$ & $6(14.0)$ & \\
\hline Ever-smoker & $44(72.1)$ & $37(86.0)$ & \\
\hline Tumor subsite & & & 1.0 \\
\hline Tonsil & $49(61.2)$ & $32(60.4)$ & \\
\hline Base of tongue & $31(38.8)$ & $21(39.6)$ & \\
\hline p16 & & & .8589 \\
\hline Positive & $45(56.2)$ & $31(58.4)$ & \\
\hline Negative & 35 (43.8) & $22(41.5)$ & \\
\hline T class $^{c}$ & & & .9712 \\
\hline $\mathrm{T} 1$ & $24(30.0)$ & $17(32.7)$ & \\
\hline $\mathrm{T} 2$ & $35(43.8)$ & $21(40.4)$ & \\
\hline T3 & $14(17.5)$ & $10(19.2)$ & \\
\hline $\mathrm{T} 4$ & $7(8.7)$ & $4(7.7)$ & \\
\hline $\mathrm{N}$ class & & & .0016 \\
\hline N1 & $10(12.5)$ & $20(37.7)$ & \\
\hline N2 & $63(78.8)$ & $32(60.4)$ & \\
\hline N3 & $7(8.7)$ & $1(1.9)$ & \\
\hline Margin status $^{d}$ & & & 1.0 \\
\hline Positive & $12(15.6)$ & $7(14.0)$ & \\
\hline Negative & $65(84.4)$ & $43(86.0)$ & \\
\hline $\begin{array}{l}\text { No. of positive lymph } \\
\text { nodes, mean (range) }\end{array}$ & $4.2(1-21)$ & $2.3(1-8)$ & .0008 \\
\hline AJCC stage & & & .0018 \\
\hline III & $9(11.2)$ & $18(34.6)$ & \\
\hline IV & $71(88.8)$ & $34(65.4)$ & \\
\hline Radiation $^{f}$ & & & .0092 \\
\hline Yes & $72(94.7)$ & $39(78.0)$ & \\
\hline No & $4(5.3)$ & $11(22.0)$ & \\
\hline Chemotherapy ${ }^{g}$ & & & .0058 \\
\hline Yes & $31(42.5)$ & $9(18.0)$ & \\
\hline No & $42(57.5)$ & $41(82.0)$ & \\
\hline
\end{tabular}

Data are presented as no. (\%) unless noted otherwise. Percentages are based on complete cases.

Abbreviations: AJCC, American Joint Committee on Cancer; ECS, extracapsular spread.

a Oropharynx patients with $<2$ months of follow-up were excluded.

${ }^{\mathrm{b}}$ Nineteen ECS-positive patients and 10 ECS-negative patients had unknown smoking status.

${ }^{\mathrm{c}}$ One ECS-negative patient had unknown T class.

dThree ECS-positive and 3 ECS-negative cases with unknown margin status.

${ }^{e}$ One ECS-negative patient had unknown AJCC stage.

${ }^{f}$ Four ECS-positive patients and 3 ECS-negative patients had unknown radiation status.

${ }^{g}$ Seven ECS-positive and 3 ECS-negative patients had unknown chemotherapy status. All patients who underwent both chemotherapy and radiation therapy did so concurrently.

who are alive with disease. Among the 76 p16-positive patients, 6 were negative for HPV as determined via in situ hybridization. Conversely, among the 57 p16-negative patients, only 1 was positive for HPV as determined via in situ hybridization.

On univariate analysis, ECS was unrelated to DSS for all OPC patients $(P=.5237)$. ECS did not correlate with DSS in patients with p16-positive $(P=.936)$ (Fig. $1 \mathrm{~A})$ nor p16-negative tumors $(P=.198)$ (Fig. 1B). ECS correlated strongly with the number of positive lymph nodes. The mean number of positive lymph nodes was 1.7 for ECS-negative cases and 5.0 for ECS-positive cases (Wilcoxon $P<.0001$ ). Furthermore, the number of positive lymph nodes was the strongest individual predictor of DSS $(P<.0001)$. Accordingly, any apparent difference in DSS in p16-negative patients (as seen in Fig. 1B) was due to confounding with the number of positive lymph nodes. After adjusting for number of positive nodes, the estimated DSS survival functions for p16-negative OPC patients, which appeared to separate from each other over time, were essentially identical (Fig. 1C).

In addition to number of positive nodes, pathological T class impacted DSS $(P=.0001)$. Adjusting for pathological T class did not alter the result that ECS has no bearing on DSS in either p16-positive or p16-negative OPC patients. Although the decision to treat OPC patients with CRT or radiotherapy (RT) was influenced by ECS status (odds ratio [OR] for CRT, 2.69; $P=.037$; OR for RT, 5.01; $P=.0092)$, neither adjuvant CRT (hazard ratio [HR], 0.87; 95\% CI, 0.41-1.81) nor RT (HR, 1.28; 95\% CI, 0.44-3.74) yielded improvement in DSS in the OPC cohort.

\section{Oral Cavity Cancer and ECS}

There were 214 patients with oral cavity cancer with cervical lymph node metastases and known ECS status. As mentioned earlier, we assumed this was a p16-negative population. The probability of 3-year DSS was much worse for patients with ECS than for those without ECS (45\% versus $71 \%$; $P=.0018$ ) (Fig. 2A). To test whether this effect was independent, we adjusted for the number of positive lymph nodes, analogous to the adjustment for ECS in patients with OPC. Interestingly, ECS remained an independent predictor of worse DSS after controlling for number of positive lymph nodes (Fig. 2B) $(P=.0251)$. Adjuvant chemotherapyand to a lesser extent, RT-improved survival in the oral cavity cohort when adjusting for ECS (HR for chemotherapy, 0.52; 95\% CI, 0.31-0.92; HR for RT, 0.60; 95\% CI, 0.34-1.06).

The only other clinico-pathologic factor found to be individually associated with DSS in the oral cavity cohort was margin status. ECS was more common in positive margin cases; the OR for ECS relative to ECS-negative was 3.3 (95\% CI, 1.2-10.6; $P=.0109$ ). To verify that the impact of ECS was independent of margin status, we adjusted the effect of ECS for both number of positive 

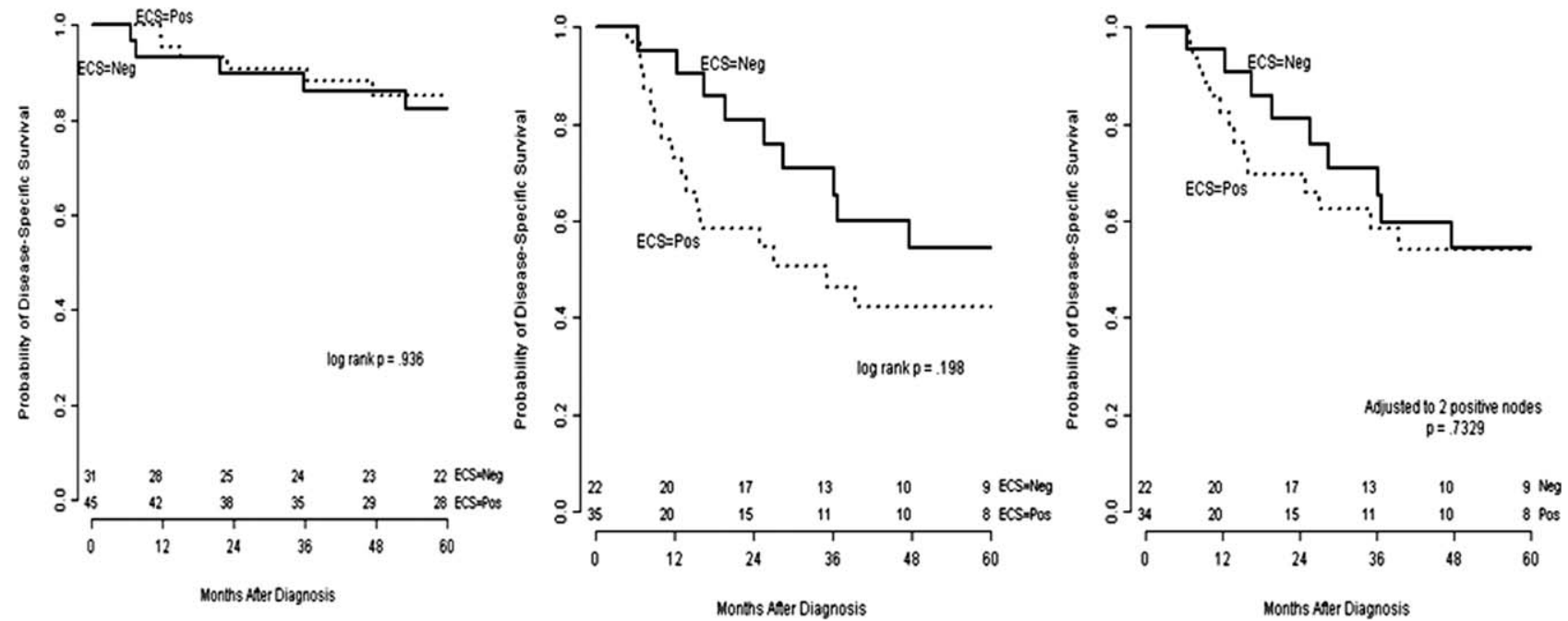

Figure 1. (A) Kaplan-Meier plot and associated log-rank test of disease-specific survival by ECS status for oropharyngeal p16positive cancer patients. (B) Kaplan-Meier plot and associated log-rank test of disease-specific survival by ECS status for oropharyngeal p16-negative cancer patients. (C) Estimated disease-specific survival by ECS among p16-negative OPC patients after adjusting for number of positive lymph nodes. Estimated survival is derived from the relative hazard function for a proportional hazards regression for patients with 2 positive nodes.
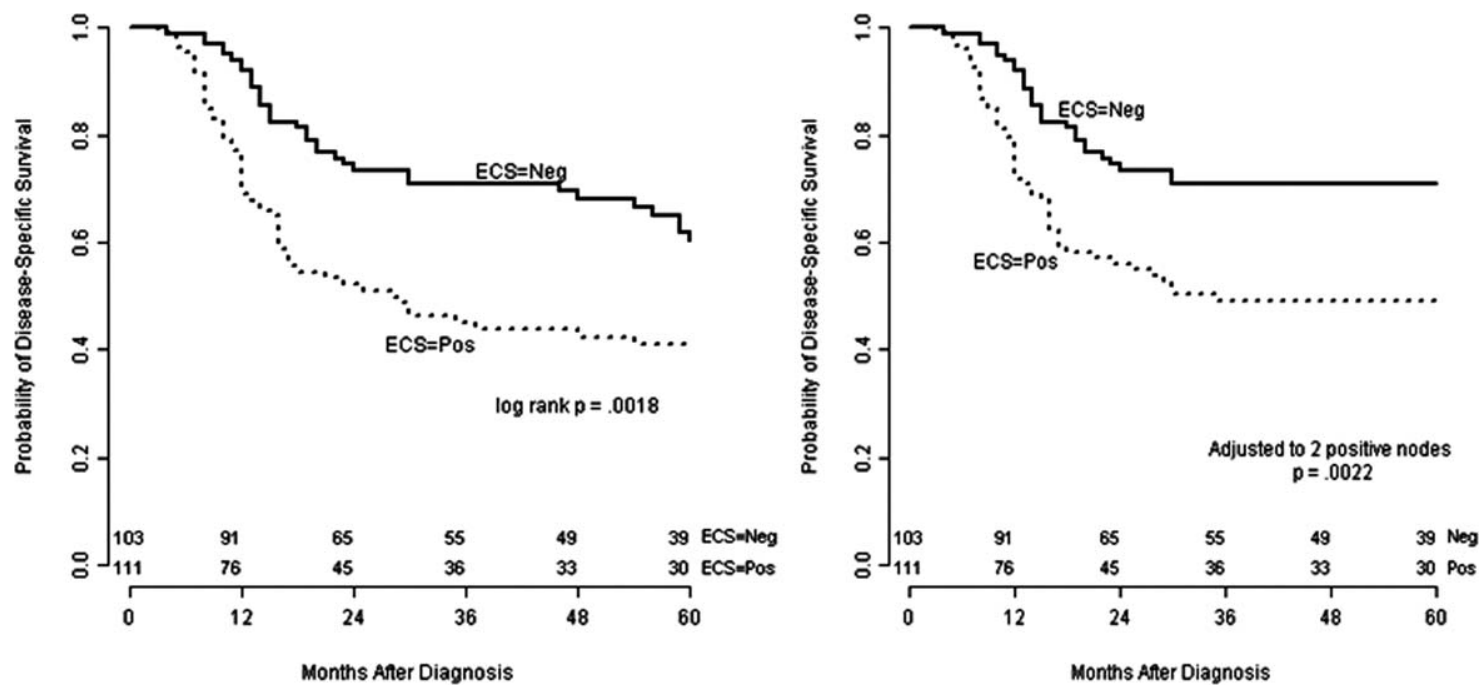

Figure 2. (A) Kaplan-Meier plot and associated log-rank test of disease-specific survival by ECS among oral cavity cancer patients. (B) Disease-specific survival by ECS in oral cavity cancer patients. Estimated survival is derived by a proportional hazard model that adjusts for number of positive lymph nodes. Due to failure to meet the assumptions of proportional hazards, interval censoring was applied. All patients surviving beyond 36 months were censored at 36 months for this analysis. Estimated survival is shown for patients with 2 positive nodes. ECS retains its prognostic ability in oral cavity cancer when adjusted for a confounding factor, whereas any apparent prognostic effect in p16-negative OPC is eliminated after adjustment for number of positive lymph nodes.

nodes and margin status. The resulting HR for ECS was 1.61 (95\% CI, 2.04-2.50; $P=.0329)$, confirming that the adverse effect of ECS on DSS remained significant and independent.

\section{DISCUSSION}

ECS is widely regarded as a pathologic marker of poor prognosis among head and neck squamous cell carcinoma patients. ${ }^{1,4,5,10,26}$ ECS and positive resection margins 
were agreed upon as the 2 major high-risk factors that warranted the use of adjuvant chemotherapy in addition to RT among advanced head and neck cancer patients. ${ }^{27-}$

${ }^{29}$ However, because nonsurgical primary management increased in popularity after the laryngeal Radiation Therapy Oncology Group (RTOG) $91-11$ trial, ${ }^{30}$ the treatment of OPC shifted from primary surgical management to up-front CRT. Due to the lack of histopathologic specimens, there has been a paucity of literature on ECS in OPC. The few studies that have examined the role of ECS in OPC had small cohorts or combined the oropharynx with other subsites, such as the oral cavity. ${ }^{12-14}$ Notably, the 2 largest prospective controlled trials identifying ECS as a negative prognostic factor had substantial proportions of OPC patients ( $42 \%$ in the RTOG trial and $30 \%$ in the European Organisation for Research and Treatment of Cancer trial). ${ }^{28,29}$ However, they did not control for primary tumor subsite, HPV status was not determined, and the prevalence of HPV was presumably lower at that time. This is the first study to our knowledge that directly compares the prognostic effect of ECS in SCCs of the oropharynx and oral cavity while controlling for primary treatment modality and HPV status of OPC.

Recently, it has been shown that ECS is not associated with poorer survival in p16-positive OPC patients. ${ }^{19,20}$ However, these studies were limited to HPV-positive OPC patients and lacked a positive control for the ECS reporting approach. Our study confirms the recent findings that ECS is not an adverse prognostic factor in HPV-positive OPC. We furthermore demonstrate that ECS remains an independent predictor of poor survival in oral cavity cancer patients. Interestingly, our findings show that ECS in HPVnegative OPC patients may appear to be marginally associated with DSS; however, any apparent adverse effect of ECS is completely explained by controlling for the number of positive lymph nodes. Therefore, the decision to treat HPVnegative OPC patients with adjuvant therapy should perhaps be based on factors other than ECS, such as number of positive lymph nodes.

A limitation inherent in this study design is the potential influence of adjuvant therapy among OPC patients. Patients with ECS-positive OPC were more likely to undergo adjuvant CRT compared with ECS-negative patients. Although the decision to treat OPC patients with CRT was influenced by ECS, neither adjuvant CRT nor RT yielded improvement in DSS in the OPC cohort. Therefore, we conclude that the lack of differential survival using ECS status in the 2 cohorts cannot be explained by patterns of adjuvant therapy. Similarly, margin status did not influence DSS in our OPC cohort, which may be due to its use as a selection criterion for adjuvant therapy. Because the number of patients with positive margins is so small, however, the results must be interpreted cautiously.

Both an important element and a limitation of this study is that our data span 3 decades (1983-2009). This extensive time frame allowed for longer follow-up and increased the sample size (especially the number of HPVnegative OPC cases). Also, because patients with OPC in the last 2 decades were often treated primarily with CRT as opposed to surgery, we needed to expand our time period in order to obtain an adequate sample size of patients treated with primary surgery. However, spanning 3 decades inevitably leads to variability in types of surgery and the dose and timing of adjuvant CRT. Because of the reemergence of trans-oral robotic surgery and laser microsurgery in the management of OPC, determining pathologic markers that help to predict prognosis is critical. Surgery traditionally provides important pathological data, such as accurate tumor staging, presence of ECS, and margin status. Based on our findings, ECS in cervical lymph node metastases among OPC patients is not indicative of poor prognosis. Future prospective validation of this finding could ultimately dismiss ECS as an adjuvant therapy selection criterion among OPC patients. As expected, ECS is an independent adverse predictor in oral cavity cancer patients, supporting the use of ECS in postsurgical patient management. Ultimately, prospective trials are warranted to further elucidate the role of pathological biomarkers, other than ECS, in predicting survival outcomes among $\mathrm{HPV}$-positive and HPV-negative OPC patients.

\section{FUNDING SOURCES}

No specific funding was disclosed.

\section{CONFLICT OF INTEREST DISCLOSURES}

The authors made no disclosures.

\section{REFERENCES}

1. Johnson JT, Barnes EL, Myers EN, Schramm VL Jr, Borochovitz D, Sigler BA. The extracapsular spread of tumors in cervical node metastasis. Arch Otolaryngol. 1981;107:725-729.

2. Ferlito A, Rinaldo A, Devaney KO, et al. Prognostic significance of microscopic and macroscopic extracapsular spread from metastatic tumor in the cervical lymph nodes. Oral Oncol. 2002;38:747-751.

3. Carter RL, Barr L.C, O’Brien CJ, Soo KC, Shaw HJ. Transcapsular spread of metastatic squamous cell carcinoma from cervical lymph nodes. Am J Surg. 1985(150):495-499.

4. Alvi A, Johnson JT. Development of distant metastasis after treatment of advanced-stage head and neck cancer. Head Neck. 1997; 19:500-505.

5. Myers JN, Greenberg JS, Mo V, Roberts D. Extracapsular spread. A significant predictor of treatment failure in patients with squamous cell carcinoma of the tongue. Cancer. 2001;92:3030-3036. 
6. Vaidya AM, Petruzzelli GJ, Clark J, Emami B. Patterns of spread in recurrent head and neck squamous cell carcinoma. Otolaryngol Head Neck Surg. 2001;125:393-396.

7. Greenberg JS, Fowler R, Gomez J, et al. Extent of extracapsular spread: a critical prognosticator in oral tongue cancer. Cancer. 2003;97:1464-1470.

8. Jan JC, Hsu WH, Liu SA, et al. Prognostic factors in patients with buccal squamous cell carcinoma: 10-year experience. J Oral Maxillofac Surg. 2011;69:396-404.

9. Shaw RJ, Lowe D, Woolgar JA, et al. Extracapsular spread in oral squamous cell carcinoma. Head Neck. 2010;32:714-722.

10. Myers EN, Alvi A. Management of carcinoma of the supraglottic larynx: evolution, current concepts, and future trends. Laryngoscope. 1996; 106:559-567.

11. Liu B, Guan C, Ji WY, Pan ZM. Extracapsular spread in ipsilateral neck metastasis: an important prognostic factor in laryngeal cancer. Chin Med Sci J. 2006;21:86-89.

12. Wenzel S, Sagowski C, Kehrl W, Metternich FU. The prognostic impact of metastatic pattern of lymph nodes in patients with ora and oropharyngeal squamous cell carcinomas. Eur Arch Otorhinolaryngol. 2004;261:270-275.

13. Klozar J, Kratochvil V, Salakova M, et al. HPV status and regional metastasis in the prognosis of oral and oropharyngeal cancer. Eur Arch Otorhinolaryngol. 2008;265(suppl 1):S75-S82.

14. Shimizu K, Inoue H, Saitoh M, et al. Distribution and impact of lymph node metastases in oropharyngeal cancer. Acta Otolaryngol. 2006;126:872-877.

15. Gillison ML, D'Souza G, Westra W, et al. Distinct risk factor profiles for human papillomavirus type 16-positive and human papillomavirus type 16-negative head and neck cancers. J Natl Cancer Inst. 2008;100:407-420.

16. Maxwell JH, Kumar B, Feng FY, et al. Tobacco use in human papillomavirus-positive advanced oropharynx cancer patients related to increased risk of distant metastases and tumor recurrence. Clin Cancer Res. 2010;16:1226-1235.

17. Fakhry C, Westra WH, Li S, et al. Improved survival of patients with human papillomavirus-positive head and neck squamous cell carcinoma in a prospective clinical trial. J Natl Cancer Inst. 2008;100:261-269.
18. Ang KK, Harris J, Wheeler R, et al. Human papillomavirus and survival of patients with oropharyngeal cancer. $N$ Engl J Med. 2010;363:24-35.

19. Lewis JS Jr, Carpenter DH, Thorstad WL, Zhang Q, Haughey BH. Extracapsular extension is a poor predictor of disease recurrence in surgically treated oropharyngeal squamous cell carcinoma. Mod Pathol. 2011;24:1413-1420.

20. Sinha P, Lewis JS Jr, Piccirillo JF, Kallogjeri D, Haughey BH. Extracapsular spread and adjuvant therapy in human papillomavirus-related, p16-positive oropharyngeal carcinoma. Cancer. 2012;118:3519-3530.

21. Chaturvedi AK, Engels EA, Pfeiffer RM, et al. Human papillomavirus and rising oropharyngeal cancer incidence in the United States. $J$ Clin Oncol. 2011;29:4294-4301.

22. Chenevert J, Chiosea S. Incidence of human papillomavirus in oropharyngeal squamous cell carcinomas: now and 50 years ago. Hum Pathol. 2012;43:17-22.

23. Edge SB, Byrd DR, Compton CC, et al, eds. AJCC Cancer Staging Manual. 7th ed. New York, NY: Springer; 2010.

24. Singhi AD, Westra WH. Comparison of human papillomavirus in situ hybridization and p16 immunohistochemistry in the detection of human papillomavirus-associated head and neck cancer based on a prospective clinical experience. Cancer. 2010;116:2166-2173.

25. D'Souza G, Dempsey A. The role of HPV in head and neck cancer and review of the HPV vaccine. Prev Med. 2011;53(suppl 1):S5-S11.

26. Johnson JT, Myers EN, Bedetti CD, Barnes EL, Schramm VL Jr, Thearle PB. Cervical lymph node metastases. Incidence and implications of extracapsular carcinoma. Arch Otolaryngol. 1985;111:534-537.

27. Bernier J, Cooper JS, Pajak TF, et al. Defining risk levels in locally advanced head and neck cancers: a comparative analysis of concurrent postoperative radiation plus chemotherapy trials of the EORTC (\#22931) and RTOG (\# 9501). Head Neck. 2005;27:843-850.

28. Cooper JS, Pajak TF, Forastiere AA, et al. Postoperative concurrent radiotherapy and chemotherapy for high-risk squamous-cell carcinoma of the head and neck. $N$ Engl J Med. 2004;350:1937-1944.

29. Bernier J, Domenge C, Ozsahin M, et al. Postoperative irradiation with or without concomitant chemotherapy for locally advanced head and neck cancer. N Engl J Med. 2004;350:1945-1952.

30. Forastiere AA, Goepfert H, Maor M, et al. Concurrent chemotherapy and radiotherapy for organ preservation in advanced laryngeal cancer. N Engl J Med. 2003;349:2091-2098. 